\title{
Encouragement of Collaborative Learning Based on Dynamic Groups
}

\author{
Ivan Srba \\ Institute of Informatics and Software Engineering \\ Faculty of Informatics and Information Technologies \\ Slovak University of Technology in Bratislava \\ Ilkovičova 3, 84216 Bratislava, Slovakia \\ srbalfit.stuba.sk
}

\begin{abstract}
Nowadays, collaboration between users is present in many web applications. This trend causes that we have to face many new challenges. One of them is group composition which plays very important role and can significantly influence the process of collaboration. In spite of many existing methods which solve group formation problem, there are a lot of unresearched possibilities how to support effective collaboration. We focused on one of them, namely how to create successful small groups iteratively and automatically without students' participation. We proposed a method which employs Group Technology optimization approach to create appropriate assignment of users to groups. We evaluated our method in educational domain in a collaborative environment called PopCorm.
\end{abstract}

\section{Categories and Subject Descriptors}

K.3.1 [Computers and Education]: Computer Uses in Education-Collaborative learning; H.5.3 [Information Interfaces and Presentation]: Group and Organization Interfaces-Asynchronous interaction

\section{Keywords}

Groups, Collaboration, Group Technology, Learning

\section{Introduction}

Web was created to support collaboration between users over the whole world. However, it lasted a long time until its position changed from static data provider to real collaboration mediator. Modern web technologies provide a

\footnotetext{
* Master degree study programme in field Software Engineering. Supervisor: Prof. Mária Bieliková, Institute of Informatics and Software Engineering, Faculty of Informatics and Information Technologies, STU in Bratislava.

(C) Copyright 2012. All rights reserved. Permission to make digital or hard copies of part or all of this work for personal or classroom use is granted without fee provided that copies are not made or distributed for profit or commercial advantage and that copies show this notice on the first page or initial screen of a display along with the full citation. Copyrights for components of this work owned by others than ACM must be honored. Abstracting with credit is permitted. To copy otherwise, to republish, to post on servers, to redistribute to lists, or to use any component of this work in other works requires prior specific permission and/or a fee. Permissions may be requested from STU Press, Vazovova 5, 81107 Bratislava, Slovakia.

Srba, I. Encouragement of Collaborative Learning Based on Dynamic Groups. Information Sciences and Technologies Bulletin of the ACM Slovakia, Special Section on the ACM Student Project of the Year 2012 Competition, Vol. 4, No. 4 (2012) 55-56
}

lot of new possibilities how to design and develop new information systems for supporting collaboration. However, users are very different in their goals and activities they use to achieve these goals. Thus their collaboration is not very successful in many cases. Therefore these systems represent new possibilities for research how to support successful and effective collaboration [5].

Collaboration in groups depends on many attributes [1]. One of the most important one is assignment of users to groups. There are many existing methods which solve this problem (e.g. jigsaw method or agent-based systems). Despite this fact, there are a lot of domains where these methods are not sufficient. First, they are not universal enough. In addition, they are static and do not consider user's actual context and are limited in considering different information sources about users. Lastly, they suppose that it is possible to decide which aspects make collaboration really effective and successful. However, this information is not well known in the current state of research. We focus on educational domain where the group composition can significantly influence collaboration.

\section{Method for creating dynamic groups}

Based on analysis of different approaches to group formation problem we proposed a method which automatically creates small short-term dynamic groups. Our method can consider any personal or collaborative user's characteristics. Collaborative characteristics can describe students' behaviour during collaboration process or relationships between students which can be derived from existing user models or interactions on social networks [3].

Our method [4] is inspired by the optimization approach called Group Technology (GT) [2]. GT approach is rooted in optimization in industry area and solves the problem how to effectively produce different parts by a set of machines. This problem can be adapted to our educational domain: we have students instead of machines and students' characteristics instead of parts.

We applied our method to the same set of students iteratively. The collaboration process is evaluated after each group finishes solving the particular task. This method's feature allows us to continually learn which characteristics should be combined together according to evaluation of how effective and successful collaboration was achieved. In addition, it is possible to automatically learn students' collaborative characteristics. 
Figure 1: The screenshot of the collabrative environment PopCorm. The categorizer tool is displayed.

Table 1: Comparison of achieved results during the second phase of the experiment

\begin{tabular}{|l|l|l|}
\hline Groups created & Avg. evaluation & Feedback \\
\hline By the proposed method & 0.459 & 4.01 \\
By the reference method & 0.392 & 3.55 \\
Randomly & 0.422 & 3.29 \\
\hline
\end{tabular}

\section{Collaborative Environment PopCorm}

We are aware that the group composition problem cannot be treated without its application in the real collaborative environment. We focus on the education at the standard one-term university course related to IT domain. Therefore we designed the set of collaborative tasks and collaborative tools which are suitable to solve these tasks. Collaborative tasks can be of several types (e.g. group discussion, advantages/disadvantages, pros/cons) where each type can be solved with one or more of available tools: a text editor, a graphical editor, a categorizer or a semi-structured discussion. The categorizer is a tool which can be used to solve tasks which solution consists of one or more lists of items. The semi-structured discussion is dedicated to communication which is independent on particular task which is solved.

We decided to employ only collaborative characteristics in group formation process. As we mentioned before, it is possible to learn these characteristics automatically due to iterative approach to group formation. Collaborative characteristic represents activities which are typically used by students to solve the assigned tasks. Examples of these activities are: propose better solution, ask for explanation or provide explanation.

In addition, we designed an approach how to automatically analyse effectiveness and successfulness of collaboration. We based this analysis on psychological studies which recommend monitoring 7 dimensions of collaboration. We added one more dimension which represents teacher's evaluation of correctness of achieved results.

We implemented the collaborative environment Popular Collaborative Platform - PopCorm according to the proposed application of our method.

\section{Evaluation and Conclusion}

We evaluated the proposed method in two steps. In the first one, we evaluated the preconditions of method. In the second one we performed a long term experiment where we compared the collaboration between groups created by our method and groups created by a reference method. As the reference method we employed k-means clustering approach. About 110 participants were iteratively assigned to 254 groups. Our method achieved better results in comparison with the reference method (see Table 1). We can consider achieved results as highly significant (with p-value about 0.0048 according to the statistical model ANOVA).

In conclusion, we proposed the method for creating dynamic short-term groups according to user's personal and collaborative characteristics. Totally 110 students actively participated on the long-term experiment and provide positive feedback about new opportunities to learn by collaboration in small groups. In addition, achieved results prove that our method created really successful and effective groups. The method and collaboration environment provide a lot of new opportunities how to support the effective collaboration in many other dimensions.

Acknowledgements. This work was partially supported by the Scientific Grant Agency of Slovak Republic, grant No. VG1/0675/11.

\section{References}

[1] T. Daradoumis, M. Guitert, F. Gimenez, J. Marques, and T. Lloret. Supporting the composition of effective virtual groups for collaborative learning. In International Conference on Computers in Education, 2002. Proceedings., volume 1, pages 332-336. IEEE Comput. Soc, 2002.

[2] H. M. Selim, R. G. Askin, and A. J. Vakharia. Cell formation in group technology: Review, evaluation and directions for future research. Comp.\& Industrial Engineering, 34(1):3-20, Jan. 1998.

[3] I. Srba and M. Bielikova. Tracing Strength of Relationships in Social Networks. In 2010 IEEE/WIC/ACM International Conference on Web Intelligence and Intelligent Agent Technology, pages 13-16. IEEE, Aug. 2010.

[4] I. Srba and M. Bieliková. Encouragement of Collaborative Learning Based on Dynamic Groups. In A. Ravenscroft, S. Lindstaedt, C. D. Kloos, and D. Hernández-Leo, editors, Lecture Notes in Computer Science, volume 7563/2012, pages 432-437. Springer Berlin / Heidelberg, 2012.

[5] G. Stahl, T. Koschmann, and D. Suthers. Computer-supported collaborative learning: An historical perspective. Cambridge handbook of the learning sciences, pages 409-426, 2006. 